九州大学学術情報リポジトリ

Kyushu University Institutional Repository

\title{
ON CERTAIN APPROXIMATIONS OF POWER OF A TEST PROCEDURE USING TWO PRELIMINARY TESTS IN A MIXED MODEL
}

Ali, M. A.

Department of Mathematics and Statistics, J. N. Agricultural University

Srivastava, S. R.

Department of Mathematics and Statistics, Banaras Hindu University

https://doi.org/10.5109/13151

出版情報: 統計数理研究. 19 (3/4)，pp. 103-113，1981-03. Research Association of Statistical Sciences

バージョン：

権利関係 : 


\title{
ON CERTAIN APPROXIMATIONS OF POWER OF A TEST PROCEDURE USING TWO PRELIMINARY TESTS IN A MIXED MODEL
}

\author{
By
}

\author{
M. A. Ali* and S. R. Srivastava**
}

(Received November 15, 1980)

\begin{abstract}
The present paper is concerned with the derivation of approximate formulae for power components of a sometimes pool test procedure applied to a mixed model experiment. A comparison of the values of power components evaluated by these formulae with those calculated using series formulae has been made.
\end{abstract}

\section{Introduction.}

In making inferences from the experimental design models, at times there may arise some doubt regarding the inclusion or not of some of the parameters in the model. For example, in a factorial experiment or an experiment with crossed classification the experimenter may be uncertain as to whether interaction parameter(s) should appear in the model. This uncertainty in the model specification may be due to the lack of knowledge, either theoretical or from past experience, in regard to the interaction effect(s) at issue. Such situations of uncertainty lead to conditional specification of the model and are to be resolved first before making final inferences.

The present study has been made for a mixed model split-plot in time experiment involving conditional specification. We are here mainly interested in making inferences regarding the split-plot treatments (split by time). The uncertainty concerning the inclusion or not of the interactions in this model has been resolved by preliminary tests of significance.

\subsection{Related Papers and Objective of the Study.}

Bozivich, Bancroft and Hartley (1956) have derived approximate formulae and exact formulae for power components of a test procedure in a component of variance model. Derivation of approximate formulae for power in a mixed model has been given by

* Department of Mathematics and Statistics, J. N. Agricultural University, Jabalpur

** Department of Mathematics and Statistics, Banaras Hindu University 
Tailor and Saxena (1974). In both of these studies, the experiments are hierarchal in nature. In the present investigation we have considered a mixed model split-plot in time experiment. This design has frequent use in experiments on forage crops [Steel and Torrie (1960)]. The object of the present study is to derive expressions for the approximate formulae of the power components of the test used and to examine the degree of accuracy of these approximations.

\subsection{Statement of the Problem}

An experiment on a certain forage crop is conducted to investigate the cutting effects with a split-plot in time layout involving ' $r$ ' blocks, ' $s$ ' varieties and ' $t$ ' cuttings. Here varieties and cuttings are fixed effects and blocks are random efiects. Let the four independent mean squares comprising cuttings be represented by $V_{1}, V_{2}, V_{3}$ and $V_{4}$ based on $n_{1}, n_{2}, n_{3}$ and $n_{4}$ degrees of freedom respectively. We are interested in testing the null hypothesis $H_{0}: E\left(V_{4}\right)=E\left(V_{3}\right)$ against the alternative $H_{1}: E\left(V_{4}\right)>E\left(V_{3}\right)$ when it is doubtful that $V_{3}$ and/or $V_{2}$ may have the same expectation as $V_{1}$. If it is certain that $E\left(V_{3}\right)$ and/or $E\left(V_{2}\right)$ is not equal to $E\left(V_{1}\right)$, the usual procedure for testing $H_{0}$ is to compare $V_{4}$ with $V_{3}$ by the $F$-statistic and to reject $H_{0}$ whenever the observed $F$-value turns out to be significant. However, if the uncertainty exists in which case $E\left(V_{3}\right)$ and/or $E\left(V_{2}\right) \geqq E\left(V_{1}\right)$, different test statistic(s) for testing $H_{0}$ may then be used by considering appropriate combination(s) of $V_{3}$ and/or $V_{2}$ with $V_{1}$. The appropriateness of these combinations may be decided by making preliminary tests for the said uncertainties.

Before we describe the proposed sometimes pool test procedure for testing $H_{0}$ and present derivation of approximate formulae for power components of this test, we state the problem precisely in the next section.

\subsection{A Precise Formulation of the Problem.}

Let us consider the following mixed model for a split-plot in time experiment

$$
\begin{gathered}
Y_{i j k}=\mu+\alpha_{i}+\beta_{j}+\delta_{i j}+\gamma_{k}+(\alpha \gamma)_{i k}+(\beta \gamma)_{j k}+\varepsilon_{i j k}, \\
i=1,2, \cdots, r \quad j=1,2, \cdots, s \quad k=1,2, \cdots, t
\end{gathered}
$$

where

$Y_{i j k}=$ yield in the $i$-th block on the $j$-th veriety with $k$-th cutting,

$\mu=$ true mean effect,

$\alpha_{i}=$ true effect of the $i$-th block,

$\beta_{j}=$ true effect of the $j$-th variety,

$\delta_{i j}=$ true effect of the experimental unit in the $i$-th block subjected to the $j$-th variety,

$\gamma_{k}=$ true effect of the $k$-th cutting,

$(\alpha \gamma)_{i k}=$ true effect of the interaction between the $i$-th block and the $k$-th cutting,

$(\beta \gamma)_{j k}=$ true effect of the interaction between the $j$-th variety and the $k$-th cutting,

$\varepsilon_{i j k}=$ true effect of the $k$-th cutting subjected to the $(i j)$-th treatment combination; and also

$$
\alpha_{i} \text { are } \operatorname{NID}\left(0, \sigma_{\alpha}^{2}\right),
$$




$$
\begin{aligned}
& \sum_{j} \beta_{j}=0, \quad \sum_{k} \gamma_{k}=0, \quad \sum_{j}(\hat{j} \gamma)_{j k}=\sum_{k}(\beta \gamma)_{j k}=0, \\
& (\alpha \gamma)_{i k} \text { are } \operatorname{NID}\left(0, \sigma_{u \gamma}^{2}\right), \quad \sum_{k}(\alpha \gamma)_{i k}=0, \quad \sum_{i}(\alpha \gamma)_{i k} \neq 0, \\
& \delta_{i j} \text { are } \operatorname{NID}\left(0, \sigma_{\delta}^{2}\right), \\
& \varepsilon_{i j k} \text { are } \operatorname{NID}\left(0, \sigma_{\varepsilon}^{2}\right) .
\end{aligned}
$$

\begin{tabular}{|c|c|c|}
\hline Source of variation & Degrees of Freedom & Expected Mean Square \\
\hline Blocks & $r-1$ & $\sigma_{\varepsilon}^{2}+s t \sigma_{a}^{\frac{2}{a}}$ \\
\hline Varieties & $s-1$ & $\sigma_{\varepsilon}^{2}+t \sigma_{\delta}^{2}+r t_{-}^{-} \sigma_{i}^{2-}$ \\
\hline Error $(a)$ & $(r-1)(s-1)$ & $\sigma_{\varepsilon}^{2}+t \sigma_{\hat{\delta}}^{2}$ \\
\hline Cuttings & $t-1$ & $\sigma_{\varepsilon}^{2}+s \sigma_{\alpha \gamma}^{2}+r s_{L_{-}^{-2}}^{-2^{-}}$ \\
\hline Cuttings $\times$ blocks & $(r-1)(t-1)$ & $\sigma_{\varepsilon}^{2}+s \sigma_{a r}^{2}$ \\
\hline Cuttings $\times$ varieties & $(s-1)(t-1)$ & $\sigma_{\varepsilon}^{2}+r_{-}^{2} \sigma_{3 r}^{2}$ \\
\hline Error (b) & $(r-1)(s-1)(t-1)$ & $\sigma_{\varepsilon}^{z}$ \\
\hline
\end{tabular}

The resulting 'anova' corresponding to (1.3.1) is given as follows:.

Table 1.1. Mixed Model ANOVA for a Split-plot in Time Experiment

where $\sigma_{\tilde{\beta}}^{2}, \sigma_{\gamma}^{2}$ and $\sigma_{\bar{\beta}}^{2}$ enclosed within parenthesis refer to finite population variances and equal $\sum_{j} \beta_{j / s-1}^{2}, \sum_{k} \gamma_{k / t-1}^{2}$ and

$$
\sum_{j} \sum_{k}(\beta \gamma)_{j k}^{2} /(s-1)(t-1)
$$

\begin{tabular}{|c|c|c|c|c|c|}
\hline \multirow{2}{*}{$\frac{\text { Source of variation }}{\text { Cuttings }}$} & \multicolumn{2}{|c|}{ Degrees of Freedom } & \multirow{2}{*}{$\frac{\text { Mean Square }}{V_{4}}$} & \multirow{2}{*}{\multicolumn{2}{|c|}{$\begin{array}{l}\text { Expected Mean Square } \\
\sigma_{\varepsilon}^{2}+s \sigma_{\alpha \gamma}^{2}+r s\left[\sigma_{\tilde{r}_{-}}^{2}=\sigma_{4}^{2}\right.\end{array}$}} \\
\hline & $t-1$ & $=n_{4}$ & & & \\
\hline Cuttings $\times$ blocks & $(r-1)(t-1)$ & $=n_{3}$ & $V_{3}$ & $\sigma_{\varepsilon}^{2}+s \sigma_{\alpha \gamma}^{2}$ & $=\sigma_{3}^{2}$ \\
\hline Cuttings $\times$ varieties & $(s-1)(t-1)$ & $=n_{2}$ & $V_{2}$ & $\sigma_{\varepsilon}^{2}+r\left[\sigma_{\beta}^{2} r_{-}^{-}\right.$ & $=\sigma_{2}^{2}$ \\
\hline Error & $(r-1)(s-1)($ & $=n_{1}$ & $V_{1}$ & $\sigma_{\varepsilon}^{2}$ & $=\sigma_{1}^{2}$ \\
\hline
\end{tabular}

respectively.

Our main interest is in whether the cuttings have any effect. We, therefore, test the hypothesis concerning $\gamma_{k}$ and confine to an abridged 'anova' as shown in Table 1.2

Table 1.2. Mixed Model Abridged ANOVA

In the above Table $1.2 V_{i}^{\prime} \mathrm{S}(i=1,3)$ are distribured as $\chi_{i}^{2} \sigma_{i}^{2} / n_{i}$, where $\chi_{i}^{2}$ is a central chi-square with $n_{i}$ degrees of freedom. On the other hand $V_{2}$ and $V_{4}$ are distributed respectively as $\chi_{2}^{\prime 2} \sigma_{1}^{2} / n_{2}$ and $\chi_{4}^{\prime 2} \sigma_{3}^{2} / n_{4}$ where $\chi_{2}^{\prime 2}$ and $\chi_{4}^{\prime 2}$ are the non-central chi-squares with $n_{2}$ and $n_{4}$ degrees of freedom and the non-centrality parameters $n_{2}\left(\sigma_{2}^{2}-\sigma_{1}^{2}\right) / 2 \sigma_{1}^{2}$ and $n_{4}\left(\sigma_{4}^{2}-\sigma_{3}^{2}\right) / 2 \sigma_{3}^{2}$.

We are interested in testing the hypothesis $H_{0}: \sigma_{4}^{2}=\sigma_{3}^{2}$ against the alternative $H_{1}: \sigma_{4}^{2}>\sigma_{3}^{2}$. From Table 1.2, it is clear that the appropriate test of $H_{0}$ is to calculate $F=V_{4} / V_{3}$ and reject $H_{0}$ if the observed value of $F$ comes out to be significant. How- 
ever, uncertainty might exist in whether $\sigma_{\alpha r}^{2}$ and or $\sigma_{\gamma i}^{2}$ is zero. In that case, we first resolve these uncertainties by making preliminary tests of significance on $\sigma_{\alpha r}^{2}$ and $\sigma_{\bar{s} r}^{2}$. Hence, we test the preliminary hypotheses $H_{01}: \sigma_{a \gamma}^{2}=0$ and $H_{02}: \sigma_{i r}^{2}=0$ in succession against their corresponding alternatives $H_{11}: \sigma_{\alpha \gamma}^{2}>0$ and $H_{12} \sigma_{\tilde{r} \gamma}^{2}>0$. The outcome of these tests are then used in devising appropriate test for $H_{0}$. It is also possible to test $\sigma_{\beta \gamma}^{2}=0$ first and then $\sigma_{a r}^{2}=0$. This may give rise to another sometimes pool test procedure which we have not studied.

The sometimes pool test procedure which we have proposed for testing $H_{0}$ is as follows :

Reject $H_{0}$ if any one of the following occurs:

$\frac{V_{3}}{V_{1}} \geqq F\left(n_{3}, n_{1} ; \alpha_{1}\right) \mathbf{l}, \quad \frac{V_{4}}{V_{3}} \geqq F\left(n_{4}, n_{3} ; \alpha_{2}\right) ;$

$$
\begin{array}{ll}
\frac{V_{3}}{V_{1}}<F\left(n_{3}, n_{1} ; \alpha_{1}\right), & \frac{V_{2}}{V_{13}} \geqq F\left(n_{2}, n_{1}+n_{3} ; \alpha_{3}\right), \\
& \frac{V_{4}}{V_{13}} \geqq F\left(n_{4}, n_{1}+n_{3} ; \alpha_{4}\right) ;
\end{array}
$$

(iii) $\frac{V_{3}}{V_{1}}<F\left(n_{3}, n_{1} ; \alpha_{1}\right)$

$$
\begin{aligned}
& \frac{V_{2}}{V_{13}}<F\left(n_{2}, n_{1}+n_{3} ; \alpha_{3}\right), \\
& \frac{V_{4}}{V_{123}} \geqq F\left(n_{4}, n_{1}+n_{2}+n_{3} ; \alpha_{5}\right) ;
\end{aligned}
$$

$$
\}
$$

where

$$
V_{13}=\frac{n_{1} V_{1}+n_{3} V_{3}}{n_{1}+n_{3}}, \quad V_{123}=\frac{n_{1} V_{1}+n_{2} V_{2}+n_{3} V_{3}}{n_{1}+n_{2}+n_{3}}
$$

and $F\left(n_{i}, n_{j} ; \alpha_{k}\right)$ refers to the upper $100 \alpha_{k} \%$ point of the F-distribution with $\left(n_{i}, n_{j}\right)$ degrees of freedom.

\section{The Power of the Proposed Sometimes Pool Test Procedure.}

The power of the sometimes pool test procedure is the sum of the probabilities associated with three mutually exclusive events given in (1.3.1). We denote these probabilities by $P_{1}, P_{2}$ and $P_{3}$, which are nothing but the power components of the test.

The derivation of series formulae for power components has heen desoribed elsewhere. The expressions for these components are as follows:

$$
\begin{aligned}
& P_{1}=A_{2} \sum_{I=0}^{\nu_{1 / 2-1}} \frac{(-1)^{I}\left(\begin{array}{c}
\nu_{4 / 2-1} \\
I
\end{array}\right)}{n_{1 / 2}+n_{3 / 2+I}} \sum_{J=0}^{I}\left(\begin{array}{l}
I \\
J
\end{array}\right) \frac{B_{x_{1}}\left(n_{1 / 2+I-J, n_{3 / 2+J}}\right)}{(1+b)^{n_{3 / 2}+J}},
\end{aligned}
$$

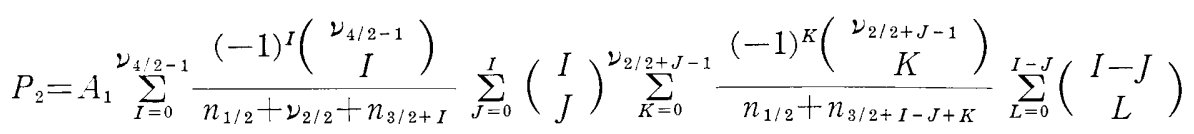

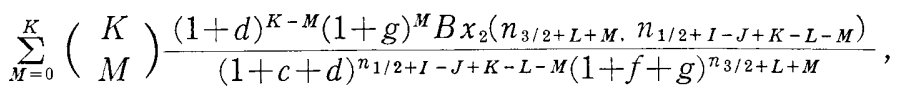




$$
\begin{aligned}
& P_{3}=-A_{1} \sum_{I=0}^{\nu_{1 / 2-1}} \frac{(-1)^{I}\left(\begin{array}{c}
\nu_{4 / 2-1} \\
I
\end{array}\right)}{n_{1 / 2}+\nu_{2 / 2}+n_{3 / 2+1}} \sum_{J=0}^{I}\left(\begin{array}{l}
I \\
J
\end{array}\right) \\
& \sum_{K=0}^{2+J-1} \frac{(-1)^{K}\left(\begin{array}{c}
\nu_{2 / 2+J-1} \\
K
\end{array}\right)}{n_{1 / 2}+n_{3 / 2+I-J+K}} \sum_{L=0}^{I-J}\left(\begin{array}{c}
I-J \\
L
\end{array}\right) \\
& \cdot \frac{1}{(1+h)^{-2 / 2+J}}\left[\frac{B_{x}\left(n_{3 / 2+L}, n_{1 / 2+I-J-L}\right)}{(1+e)^{n_{1 / 2+I}-J-L(1+m)^{n_{3 / 2+L}}}}\right.
\end{aligned}
$$

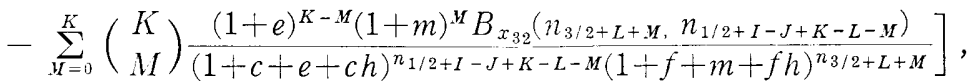

where

$$
\begin{aligned}
& A_{1}=\frac{\Gamma\left(n_{1 / 2}+\nu_{2 / 2}+n_{3 / 2}+\nu_{4 / 2}\right)}{\Gamma\left(n_{1 / 2}\right) \Gamma\left(\nu_{2 / 2}\right) \Gamma\left(n_{3 / 2}\right) \Gamma\left(\nu_{4 / 2}\right)}, \quad A_{2}=\frac{\Gamma\left(n_{1 / 2}+n_{3 / 2}+\nu_{4 / 2}\right)}{\Gamma\left(n_{1 / 2}\right) \Gamma\left(n_{3 / 2}\right) \Gamma\left(\nu_{4 / 2}\right)}, \\
& a=\frac{n_{3}}{\theta_{31} n_{1}} F\left(n_{3}, n_{1} ; \alpha_{1}\right), \quad b=-\frac{n_{4}}{c_{4} n_{3}} F\left(n_{4}, n_{3} ; \alpha_{2}\right), \\
& c=\frac{n_{2}}{c_{2}\left(n_{1}+n_{3}\right)} F\left(n_{2}, n_{1}+n_{3} ; \alpha_{3}\right), \quad d=\frac{n_{4}}{c_{4} \theta_{31}\left(n_{1}+n_{3}\right)} F\left(n_{4}, n_{1}+n_{3} ; \alpha_{4}\right), \\
& e=\frac{n_{4}}{c_{4} \theta_{31}\left(n_{1}+n_{2}+n_{3}\right)} F\left(n_{4}, n_{1}+n_{2}+n_{3} ; \alpha_{5}\right), \quad f=-\frac{\theta_{31} n_{2}}{c_{2}\left(n_{1}+n_{3}\right)} F\left(n_{2}, n_{1}+n_{3} ; \alpha_{3}\right), \\
& g=\frac{n_{4}}{c_{4}\left(n_{1}+n_{3}\right)} F\left(n_{4}, n_{1}+n_{3} ; \alpha_{4}\right), \quad h=\frac{c_{2} n_{4}}{c_{4} \theta_{31}\left(n_{1}+n_{2}+n_{3}\right)} F\left(n_{4}, n_{1}+n_{2}+n_{3} ; \alpha_{5}\right), \\
& m=\frac{n_{4}}{c_{4}\left(n_{1}+n_{2}+n_{3}\right)} F\left(n_{4}, n_{1}+n_{2}+n_{3} ; \alpha_{5}\right), \quad \theta_{31}=-\frac{\sigma_{3}^{2}}{\sigma_{1}^{2}} ; \\
& x_{1}=\frac{1}{1+a(1+b)}, \quad x_{2}=\frac{a(1+f+g)}{1+c+d+a(1+f+g)}, \\
& x_{31}=\frac{a(1+m)}{1+e+a(1+m)}, \quad x_{32}=\frac{a(1+f+m+f h)}{1+c+e+c h+a(1+f+m+f h)},
\end{aligned}
$$

We now evaluate the power components using approximations:

\subsection{Derivation of Approximate Formulae.}

Let

$$
\left.\begin{array}{l}
F_{1}=F\left(n_{3}, n_{1} ; \alpha_{1}\right) \\
F_{2}=F\left(n_{4}, n_{3} ; \alpha_{2}\right) \\
F_{3}=F\left(n_{2}, n_{1}+n_{3} ; \alpha_{3}\right) \\
F_{4}=F\left(n_{4}, n_{1}+n_{3} ; \alpha_{4}\right) \\
F_{5}=F\left(n_{4}, n_{1}+n_{2}+n_{3} ; \alpha_{5}\right)
\end{array}\right\}
$$

Then the power components $P_{1}, P_{2}, P_{3}$ can be written as 


$$
\begin{aligned}
& P_{1}: \text { Prob. }\left\{\frac{V_{3}}{V_{1}} \geqq F_{1}, \quad \frac{V_{4}}{V_{3}} \geqq F_{2}\right\}, \\
& P_{2} \text { : Prob. }\left\{\frac{V_{3}}{V_{1}}<F_{1}, \quad \frac{V_{2}}{V_{13}} \geqq F_{3}, \quad \frac{V_{4}}{V_{13}} \geqq F_{4}\right\}, \\
& P_{3} \text { : Prob. }\left\{\frac{V_{3}}{V_{1}}<F_{1}, \quad \frac{V_{2}}{V_{13}}<F_{3}, \quad \frac{V_{4}}{V_{123}} \geqq F_{5}\right\} .
\end{aligned}
$$

\subsubsection{Approximate Formula for $P_{1}$.}

To evaluate (2.1.b), we use Patnaik's (1949) approximation to non-central chi-square as well as normal approximation to $\log V_{i}$ suggested by Bartlett and Kendall (1946). The latter approximation holds good provided $n_{i}$ is not too small.

If we write

$$
\begin{aligned}
& y_{1}=\log V_{3}-\log V_{1} \\
& y_{2}=\log V_{4}-\log V_{3}
\end{aligned}
$$

then it can be shown that the joint density of $y_{1}$ and $y_{2}$ is bivariate normal with means :

$$
\begin{aligned}
& \bar{y}_{1}=\log \sigma_{3}^{2}-\log \sigma_{1}^{2}=\log \theta_{31} \\
& \bar{y}_{2}=\log \sigma_{4}^{2}-\log \sigma_{3}^{2}=\log \left(1+\frac{2 \lambda_{4}}{n_{4}}\right),
\end{aligned}
$$

variances:

$$
\begin{aligned}
& \operatorname{Var}\left(y_{1}\right)=\frac{2}{n_{3}-1}+\frac{2}{n_{1}-1} \\
& \operatorname{Var}\left(y_{2}\right)=\frac{2}{\nu_{4}-1}+\frac{2}{n_{3}-1},
\end{aligned}
$$

and the coefficient of correlation:

$$
\rho_{y_{1} y_{2}}=-\left\{\frac{\left(\nu_{4}-1\right)\left(n_{1}-1\right)}{\left(n_{3}+n_{1}-2\right)\left(\nu_{4}+n_{3}-2\right)}\right\}^{1 / 2} .
$$

Therefore, $P_{1}$ can be written as

$$
P_{1} \cong \text { Prob. }\left\{x \geqq u, y \geqq v, \rho_{y_{1} y_{2}}\right\},
$$

where $x$ and $y$ are standard normal variates and the argument $u$ and $v$ are given by

$$
\begin{aligned}
& u=\frac{2 Z\left(n_{3}, n_{1} ; \alpha_{1}\right)-\log \theta_{31}}{\sqrt{\frac{2}{n_{3}-1}+\frac{2}{n_{1}-1}}} \\
& v=\frac{2 Z\left(n_{4}, n_{3} ; \alpha_{2}\right)-\log \left(1+\frac{2 \lambda_{4}}{n_{4}}\right)}{\sqrt{\frac{2}{\nu_{4}-1}+\frac{2}{n_{3}-1}}} .
\end{aligned}
$$

$Z\left(n_{i}, n_{j} ; \alpha_{k}\right)$ denotes the upper $\approx 100 \alpha_{k} \%$ point of Fisher's Z-distribution with $\left(n_{i}, n_{j}\right)$ degrees of freedom. 
The probability as in (2.1.1.5) can now be evaluated by employing the table of probability integral of a bivariate normal surface [Published by National Bureau of Standards (1959)].

\subsubsection{Approximate Formula for $P_{2}$.}

With regard to $P_{2}$, we observe that, in the limit, the three ratios $\frac{V_{3}}{V_{1}}, \frac{V_{2}}{V_{13}}$ and $\frac{V_{4}}{V_{13}}$ are independently distributed since for finite $n_{3}$ as $n_{1} \rightarrow \infty$, (i.e. $s \rightarrow \infty$ ), $V_{1}$ and $V_{13}$ both tend to $\sigma_{1}^{2}$. Hence, (2.1.c) can be written as

$$
P_{2} \cong \text { Prob. }\left\{\frac{V_{3}}{V_{1}}<F_{1}\right\} \text { Prob. }\left\{\frac{V_{2}}{V_{13}} \geqq F_{3}\right\} \text { Prob. }\left\{\frac{V_{4}}{V_{13}} \geqq F_{4}\right\}
$$

From Patnaik's (1949) approximation we find that $n_{2} V_{2}$ and $n_{4} V_{4}$ are distributed as $\chi_{\nu_{2}}^{2} c_{2} \sigma_{1}^{2}$ and $\chi_{\nu_{4} c_{4}}^{2} c_{3}^{2}$, where $\chi_{\nu_{2}}^{2}$ and $\chi_{\nu_{4}}^{2}$ are the central chi-squares w!th degrees of freedom

$$
\nu_{2}=n_{2}+\frac{4 \lambda_{2}^{2}}{n_{2}+4 \lambda_{2}}, \quad \nu_{4}=n_{4}+\frac{4 \lambda_{4}^{2}}{n_{4}+4 \lambda_{4}}
$$

and scalar constants

$$
c_{2}=1+\frac{2 \lambda_{2}}{n_{2}+2 \lambda_{2}}, \quad c_{4}=1+\frac{2 \lambda_{4}}{n_{4}+2 \lambda_{4}}
$$

Also $n_{i} V_{i}(i=1,3)$ is distributed as $\chi_{i}^{2} \sigma_{i}^{2}$ where $\chi_{i}^{2}$ is a central chi-square with $n_{i}$ degrees of freedom and $\left(n_{1}+n_{3}\right)^{2} V_{13}$ is approximately distributed as $\left(n_{1} \sigma_{1}^{2}+n_{3} \sigma_{3}^{2}\right) \chi_{13}^{2}$, where $\gamma_{13}^{2}$ is a central chi-square with $n_{1}+n_{3}$ degrees of freedom.

Making use of these approximations, we can write (2.1.2.1) as

$$
\begin{aligned}
P_{2}= & \operatorname{Prob}\left\{F\left(n_{3}, n_{1}>\frac{F_{1}}{\theta_{31}}\right\} \operatorname{Prob} .\left\{F\left(\nu_{2}, n_{1}+n_{3}\right) \geqq \frac{n_{2}\left(n_{1}+n_{3} \theta_{31}\right) F_{3}}{\left(n_{1}+n_{3}\right)\left(n_{2}+2 \lambda_{2}\right)}\right\}\right. \\
& \operatorname{Prob}\left\{F\left(\nu_{4}, n_{1}+n_{3}\right) \geqq \frac{n_{4}\left(n_{1} \theta_{31}+n_{3}\right) F_{4}}{\left(n_{1}+n_{3}\right)\left(n_{4}+2 \lambda_{4}\right)}\right\},
\end{aligned}
$$

where $F(p, q)$ is the central $F$-statistic based on $(p, q)$ degrees of freedom.

If we now use the relation

$$
\text { Prob. }\left\{F(p, q) \leqq F_{0}\right\}=I_{x}\left(\frac{p}{2}, \frac{q}{2}\right),
$$

where $x=p F_{0} /\left(q+p F_{0}\right)$, between $F$-integral and normalized incomplete Beta function, we obtain

$$
P_{2} \cong I_{x_{21}}\left(\frac{n_{3}}{2}, \frac{n_{1}}{2}\right)\left[1-I x_{22}\left(\frac{\nu_{2}}{2}, \frac{n_{1}+n_{3}}{2}\right)\right]\left[1-I x_{23}\left(\frac{\nu_{4}}{2}, \frac{n_{1}+n_{3}}{2}\right)\right],
$$

where

$$
\begin{aligned}
& x_{21}=\frac{n_{3} F_{1}}{n_{1} \theta_{31}+n_{3} F_{1}}, \\
& x_{22}=\frac{\nu_{2} n_{2}\left(n_{1}+n_{3} \theta_{31}\right) F_{3}}{\left(n_{1}+n_{3}\right)^{2}\left(n_{2}+2 \lambda_{2}\right)+\nu_{2} n_{2}\left(n_{1}+n_{3} \theta_{31}\right) F_{3}}
\end{aligned}
$$


and

$$
x_{23}=\frac{\nu_{4} n_{4}\left(n_{1} \theta_{31}+n_{3}\right) F_{4}}{\left(n_{1}+n_{3}\right)^{2}\left(n_{4}-2 \lambda_{4}\right)+\nu_{4} n_{4}\left(n_{1} \theta_{31}-n_{3}\right) F_{4}} \cdot
$$

To evaluate the probability $P_{2}$ given by $(2.1 .2 .6)$ we can use the Tabies of Incomplete Beta Function edited by Pearson (1968).

\subsubsection{Approximate Fomula for $P_{3}$.}

To evaluate $P_{3}$, we observe that for finite $n_{3}$ if $n_{1} \rightarrow \infty$ (i. e., $s \rightarrow \infty$ ) both $V_{1}$ and $V_{13}$ tend to $\sigma_{1}^{2}$ and $V_{123}$ tends to $\frac{(r-1) \sigma_{1}^{2}}{r}+\frac{\sigma_{2}^{2}}{r}$ and therefore, in the limit the three ratios $\frac{V_{3}}{V_{1}}, \frac{V_{2}}{V_{13}}$ and $\frac{V_{4}}{V_{123}}$ are independently distributed. Hence, we may write (2.1.d) as

$$
P_{3} \cong \operatorname{Prob} .\left\{\frac{V_{3}}{V_{1}}<F_{1}\right\} \text { Prob. }\left\{\frac{V_{2}}{V_{13}}<F_{3}\right\} \text { Prob. }\left\{\frac{V_{4}}{V_{123}} \geqq F_{\overline{5}}\right\}
$$

Using Patnaik's approximation we find that $\left(n_{1}+n_{2}+n_{3}\right) V_{123}$ is distributed as $c^{\prime} \chi_{\nu^{\prime}}^{2} \sigma_{1}^{2}$, where $\chi_{\nu^{\prime}}^{2}$ is a central chisquare with degrees of freedom

$$
\nu^{\prime}=n_{1}+n_{2}+n_{3}+\frac{4 \lambda_{2}^{2}}{n_{1}+n_{2}+n_{3}+4 \lambda_{2}}
$$

and the scalar constant

$$
c^{\prime}=1+\frac{2 \lambda_{2}}{n_{1}+n_{2}+n_{3}+2 \lambda_{2}}
$$

If we use this approximation and follow the same method as in section 2.1.2, we obtain

$$
\begin{aligned}
P_{3} \cong & \operatorname{Prob} .\left\{F\left(n_{3}, n_{1}\right)<\frac{F_{1}}{\theta_{31}}\right\} \operatorname{Prob} .\left\{F\left(\nu_{2}, n_{1}+n_{3}\right)<\frac{n_{2}\left(n_{1}+n_{3} \theta_{31}\right) F_{3}}{\left(n_{1}+n_{3}\right)\left(n_{2}+2 \lambda_{2}\right)}\right\} \\
& \text { Prob. }\left\{F\left(\nu_{1}, \nu^{\prime}\right) \geqq \frac{n_{4}\left(n_{1}+n_{2}+n_{3}+2 \lambda_{2}\right) F_{5}}{\left(n_{4}+2 \lambda_{4}\right)\left(n_{1}+n_{2}+n_{3}\right) \theta_{31}}\right\}
\end{aligned}
$$

Making use of (2.1.2.5), we have

$$
P_{3} \cong I_{x_{21}}\left(\frac{n_{3}}{2}, \frac{n_{1}}{2}\right) I_{x_{22}}\left(\frac{\nu_{2}}{2}, \frac{n_{1}+n_{3}}{2}\right)\left[1-I_{x_{33}}\left(\frac{\nu_{i}}{2}, \frac{\nu^{\prime}}{2}\right)\right],
$$

where $x_{21}$ and $x_{22}$ are given by (2.1.2.7) and (2.1.2.8) respectively and

$$
x_{33}=\frac{n_{4}\left(n_{1}+n_{2}+n_{3}+2 \lambda_{2}\right) F_{5}}{\nu^{\prime}\left(n_{1}+n_{2}+n_{3}\right) \theta_{31}+n_{4}\left(n_{1}+n_{2}+n_{3}+2 \lambda_{2}\right) F_{5}} .
$$

The probability $P_{3}$ given by (2.1.3.5) can easily be computed by using Pearson's (1968) Table.

\section{Results and Discussion.}

In this section, we attempt to examine how the proposed approximations work. Using approximate formulae developed in section 2.1 we have made numerical calcui- 
ations for power components. We have also computed values for power components by applying series formulae given by (2.1), (2.2) and (2.3). In order to see how these approximations work, we have considered the values for $P_{2}$ and $P_{3}$ only obtained by both series and approximate formulae. These values along with their differences have been presented in Tables 3.1-3.4 for $\alpha_{p}=.50$ and $\alpha_{f}=.05$, where $\alpha_{p}$ and $\alpha_{f}$ are respectively the preliminary and final levels of significance. Tables 3.1 and 3.2 show the values of $P_{2}$ computed using series and approximate formulae, alongwith differences in these values for $c_{2}=1.0000$ and $c_{2}=1.7071$ respectively. Tables 3.3 and 3.4 show similar values for $P_{3}$. In all these tables we have taken $\lambda_{4}=0$.

It is observed from Tables 3.1 and 3.2 that for a given set of degrees of freedom and for $\theta_{31}=1.0$, the difference between the series and approximate values of $P_{2}$ decreases as $c_{2}$ increases from 1.0000 to 1.7071 . This difierence decreases rapidly as we increase $n_{1}$ singly or in combination with $n_{2}$ or with $n_{2}$ and $n_{3}$ and becomes nearly zero (.00008) when $n_{1}, n_{2}$ and $n_{3}$ each equals 10 . For $\theta_{31}>1.0$, this tendency is reversed. For a fixed value of degrees of freedom combination, the difference between the two values increases as $\theta_{31}$ increases and then decteases for large values of $\theta_{31}$.

From Tables 3.3 and 3.4 , it can be seen that for $\theta_{31}=1.0$ and $c_{2}=1.0000$, the approximate formula yields thesame value for $P_{3}$ as the series formula for all the sets of degrees of freedom considered. For $n_{1}=n_{2}=n_{3}=n_{4}=2$ when $c_{2}$ exceeds unity, the difference in values obtained by the two formulae differs very slightly from zero. For large values of $n_{1}, n_{2}$ and $n_{3}$, this diherence approaches zero. For a fixed value of $c_{2}$ and given degrees of freedom combination, the difference in the two values of $P_{3}$

Table 3.1. Nature of Approximation of $P_{2}$ for $n_{1}=2, \alpha_{p}=.50, \alpha_{f}=.05, \lambda_{4}=0, c_{2}=1.0000$

\begin{tabular}{|c|c|c|c|c|c|c|c|c|}
\hline \multirow{2}{*}{$n_{3}$} & \multirow{2}{*}{$n_{2}$} & \multirow{2}{*}{$\theta_{31}$} & \multicolumn{3}{|c|}{$n_{1}=2$} & \multicolumn{3}{|c|}{$n_{1}=10$} \\
\hline & & & Series & approx. & diff. & Series & approx. & diff. \\
\hline \multirow[t]{6}{*}{2} & 2 & 1.0 & .02094 & .01250 & .00844 & .01626 & .01250 & .00376 \\
\hline & & 1.5 & .02509 & .00609 & .01900 & .02556 & .00359 & .02197 \\
\hline & & 2.0 & .02736 & .00329 & .02407 & .03102 & .00124 & .02978 \\
\hline & & 3.0 & .02896 & .00118 & .02778 & .03494 & .00021 & .03473 \\
\hline & & 5.0 & .02776 & .00025 & .02751 & .03310 & .00001 & .03309 \\
\hline & & 8.0 & .02397 & .00005 & .02392 & .02720 & .00000 & .02720 \\
\hline \multirow[t]{6}{*}{2} & 10 & 1.0 & & & & .01967 & .01250 & .00717 \\
\hline & & 1.5 & & & & .02987 & .00340 & .02647 \\
\hline & & 2.0 & & & & .03522 & .00110 & .03412 \\
\hline & & 3.0 & & & & .03812 & .00017 & .03795 \\
\hline & & 5.0 & & & & .03458 & .00001 & .03457 \\
\hline & & 8.0 & & & & .02758 & .00000 & .02758 \\
\hline \multirow[t]{6}{*}{10} & 10 & 1.0 & & & & .01751 & .01250 & .00501 \\
\hline & & 1.5 & & & & .01397 & .00244 & .01153 \\
\hline & & 2.0 & & & & .00944 & .00049 & .00895 \\
\hline & & 3.0 & & & & .00402 & .00002 & .00400 \\
\hline & & 5.0 & & & & .00089 & .00000 & .00089 \\
\hline & & 8.0 & & & & .00016 & .00000 & .00016 \\
\hline
\end{tabular}


Table 3.2. Nature of Approximation of $\mathrm{P}_{2}$ for $n_{4}=2, \alpha_{p}=.50, \alpha_{f}=.05, \lambda_{4}=0, c_{2}=1.7071$

\begin{tabular}{|c|c|c|c|c|c|c|c|c|}
\hline \multirow{2}{*}{$n_{3}$} & \multirow{2}{*}{$n_{2}$} & \multirow{2}{*}{$\theta_{31}$} & \multicolumn{3}{|c|}{$n_{1}=2$} & \multicolumn{3}{|c|}{$n_{1}=10$} \\
\hline & & & Series & approx. & diff. & Series & approx. & diff. \\
\hline \multirow[t]{6}{*}{2} & 2 & 1.0 & .02481 & .02251 & .00230 & .02420 & .02312 & .00108 \\
\hline & & 1.5 & .03150 & .01210 & .01940 & .04035 & .00693 & .03342 \\
\hline & & 2.0 & .03600 & .00713 & .02887 & .05079 & .00249 & .04830 \\
\hline & & 3.0 & .04094 & .00297 & .03797 & .05975 & .00046 & .05929 \\
\hline & & 5.0 & .04310 & .00079 & .04231 & .05896 & .00004 & .05892 \\
\hline & & 8.0 & .04033 & .00019 & .04014 & .04973 & .00000 & .04973 \\
\hline \multirow[t]{6}{*}{2} & 10 & 1.0 & & & & .02499 & .02482 & .00017 \\
\hline & & 1.5 & & & & .04207 & .00750 & .03457 \\
\hline & & 2.0 & & & & .05330 & .00271 & .05059 \\
\hline & & 3.0 & & & & .06320 & .00051 & .06269 \\
\hline & & 5.0 & & & & .06286 & .00004 & .06282 \\
\hline & & 8.0 & & & & .05328 & .00000 & .05328 \\
\hline \multirow[t]{4}{*}{10} & 10 & 1.0 & & & & .02499 & .02491 & .00008 \\
\hline & & 2.0 & & & & .01968 & .00208 & .01760 \\
\hline & & 5.0 & & & & .00315 & .00000 & .00315 \\
\hline & & 8.0 & & & & .00070 & .00000 & .00070 \\
\hline
\end{tabular}

Table 3.3. Nature of Approximation of $P_{3}$ for $n_{4}=2, \alpha_{p}=.50, \alpha_{f}=.05, \lambda_{4}=0, c_{2}=1.0000$

\begin{tabular}{|c|c|c|c|c|c|c|c|c|}
\hline \multirow{2}{*}{$n_{3}$} & \multirow{2}{*}{$n_{2}$} & \multirow{2}{*}{$\theta_{31}$} & \multicolumn{3}{|c|}{$n_{1}=2$} & \multicolumn{3}{|c|}{$n_{1}=10$} \\
\hline & & & Series & approx. & diff. & Series & approx. & diff. \\
\hline \multirow[t]{6}{*}{2} & 2 & 1.0 & .01250 & .01250 & .00000 & .01250 & .01250 & .00000 \\
\hline & & 1.5 & .01881 & .02300 & -.00419 & .02182 & .02351 & -.00169 \\
\hline & & 2.0 & .02353 & .03224 & -.00871 & .02803 & .03168 & -.00365 \\
\hline & & 3.0 & .02924 & .04515 & -.01591 & .03355 & .04070 & -.00715 \\
\hline & & 5.0 & .03266 & .05514 & -.02248 & .03346 & .04500 & -.01154 \\
\hline & & 8.0 & .03114 & .05449 & -.02335 & .02834 & .04232 & -.01398 \\
\hline \multirow[t]{6}{*}{2} & 10 & 1.0 & & & & .01250 & .01250 & .00000 \\
\hline & & 1.5 & & & & .02341 & .02582 & -.00241 \\
\hline & & 2.0 & & & & .03069 & .03642 & -.00573 \\
\hline & & 3.0 & & & & .03694 & .04898 & -.01204 \\
\hline & & 5.0 & & & & .03650 & .05562 & -.01912 \\
\hline & & 8.0 & & & & .03055 & .05156 & -.02101 \\
\hline \multirow[t]{6}{*}{10} & 10 & 1.0 & & & & .01250 & .01250 & .00000 \\
\hline & & 1.5 & & & & .01636 & .02227 & -.00591 \\
\hline & & 2.0 & & & & .01497 & .02315 & -.00818 \\
\hline & & 3.0 & & & & .00912 & .01515 & -.00603 \\
\hline & & 5.0 & & & & .00287 & .00458 & -.00171 \\
\hline & & 8.0 & & & & .00065 & .00096 & -.00031 \\
\hline
\end{tabular}


Table 3.4. Nature of Approximation of $P_{3}$ for $n_{4}=2, \alpha_{p}=.50, \alpha_{f}=.05, \lambda_{4}=0, c_{2}=1.7071$

\begin{tabular}{|c|c|c|c|c|c|c|c|c|}
\hline \multirow{2}{*}{$n_{3}$} & \multirow{2}{*}{$n_{2}$} & \multirow{2}{*}{$\theta_{31}$} & \multicolumn{3}{|c|}{$n_{1}=1$} & \multicolumn{3}{|c|}{$n_{1}=10$} \\
\hline & & & Series & approx. & diff. & Series & approx. & diff. \\
\hline \multirow[t]{6}{*}{2} & 2 & 1.0 & .00074 & .00118 & -.00044 & .00112 & .00103 & .00009 \\
\hline & & 1.5 & .00150 & .00294 & -.00144 & .00230 & .00242 & -.00012 \\
\hline & & 2.0 & .00227 & .00521 & -.00294 & .00323 & .00384 & -.00061 \\
\hline & & 3.0 & .00361 & .01028 & -.00667 & .00427 & .00623 & -.00196 \\
\hline & & 5.0 & .00518 & .01907 & -.01389 & .00465 & .00941 & -.00476 \\
\hline & & 8.0 & .00592 & .02635 & -.02043 & .00416 & .01204 & -.00788 \\
\hline \multirow[t]{6}{*}{2} & 10 & 1.0 & & & & .00002 & .00001 & .00001 \\
\hline & & 1.5 & & & & .00008 & .00006 & .00002 \\
\hline & & 2.0 & & & & .00015 & .00019 & -.00004 \\
\hline & & 3.0 & & & & .00027 & .00068 & -.00041 \\
\hline & & 5.0 & & & & .00039 & .00251 & -.00212 \\
\hline & & 8.0 & & & & .00040 & .00636 & -.00596 \\
\hline \multirow[t]{4}{*}{10} & 10 & 1.0 & & & & .00002 & .00001 & .00001 \\
\hline & & 2.0 & & & & .00011 & .00031 & -.00020 \\
\hline & & 5.0 & & & & .00008 & .00105 & -.00097 \\
\hline & & 8.0 & & & & .00003 & .00050 & -.00047 \\
\hline
\end{tabular}

decreases as $\theta_{31}$ incresses and is, in general, negative.

We may, therefore, conclude that the approximate formulae for $P_{2}$ and $P_{3}$ work out satisfactorily for $\theta_{31}=1.0$ and $c_{2}>1$ (i. e., the interaction exists between cuttings and varieties). For large $\theta_{31}$ (say $\theta_{31} \geqq 8.0$ ), the proposed approximation would work provided each of $n_{1}, n_{2}$ and $n_{3}$ is greater than or equal to 10 irrespective of whether the interaction is present or not (i. e., $c_{2} \geqq 1$ ). Under these codditions, the proposed approximate formulae render considerable relief in the computation of power values.

\section{Acknowledgements.}

This research was financially supported by the ICAR fellowship.

\section{References}

[1] Bartlett, M.S. and Kendall, D. G. (1946). The statistical analysis of variance heterogeniety and the logarithmic transformation. Jour. Roy. Stat. Soc. Suppl., 8, 128-138.

[2] Bozivich, H. Baxcroft, T. A. and Hartley, H.O. (1956). Power of analysis of variance test procedure for certain incompletely specified models I. Ann. Math. Stat., 27, 1017-1043.

[3] National Buresu of Standards (1959). Tables of the Bivariate Normal Distribution Function and Related Functions. Applied Mathematics Series 50, U.S. Government Printing Office, Washington, D.C.

[4] PAtraik, P.B. (1949). The non-central $\chi^{2}$ and F-distributions and their applications. Biometrika, 36, 202-232.

[5] Pearson, K. (1968). Tables of the Incomplete Beta-Function, Second edition, Cambridge University Press.

[6] Steel, R. G.D. and Torrie, J.H. (1960). Principles and Procedures of Statistics, McGraw Hill Book Company, Inc., New York.

[7] TAILOR, J. K. and Saxena, K.P. (1974). Approximate formulae for power components of sometimes pool test procedure based on a mixed model. Sankhya, Ser. B, 36, 267-277. 